\title{
Variáveis pessoais e socioculturais de diferenciação das expectativas académicas: Estudo com alunos do Ensino Superior do Norte de Portugal e Galiza
}

\author{
Alexandra M. Araújoi \& Leandro S. Almeidaii \\ Universidade do Minho, Portugal \\ Alexandra R. Costaiii \\ Instituto Politécnico do Porto, Portugal \\ Sonia Alfonsoiv, Ángeles Condev \& Manuel Deañovi \\ Universidade de Vigo-Ourense, Espanha
}

\begin{abstract}
Resumo
Com a expansão do acesso ao Ensino Superior, nas últimas décadas assistimos a uma maior heterogeneidade de estudantes neste nível de ensino. Uma das variáveis que importa considerar na análise dos perfis de estudantes universitários são as suas expectativas académicas, uma vez que as mesmas estão associadas a níveis diferentes de envolvimento, permanência e sucesso escolar. Foi administrado um questionário de expectativas a uma amostra de 717 estudantes do $1^{\circ}$ ano, portugueses e espanhóis, avaliando sete dimensões: i) Formação para o emprego e carreira; ii) Desenvolvimento pessoal e social; iii) Mobilidade internacional; iv) Envolvimento político e cidadania; v) Pressão social; vi) Qualidade da formação; e vii) Interação social. Os resultados foram analisados em função do país, do sexo e de os estudantes serem ou não de primeira geração (se nenhum dos pais possui frequência do ES). As análises mostram que, de um modo geral, os estudantes espanhóis apresentam expectativas académicas mais baixas do que os estudantes portugueses. Por outro lado, observa-se
\end{abstract}


um efeito de sentido inverso entre ser ou não estudante de primeira geração, sendo que estudantes cujos pais possuem formação superior apresentam expectativas mais elevadas em Portugal, ocorrendo uma situação inversa com os estudantes de Espanha. Algumas explicações são avançadas na interpretação destes dados.

Palavras-chave

Ensino Superior; Expectativas académicas; Estudantes do primeiro ano; Variáveis socioculturais

\section{Introdução}

Nas últimas décadas temos assistido a uma elevada procura do Ensino Superior (ES) por parte dos jovens, que, na sua maioria, transitam diretamente do ensino secundário para uma educação superior (estudantes tradicionais). Em função desta maior procura, associada a uma maior democratização do acesso ao ES, verifica-se também uma maior diversidade de estudantes em termos das suas capacidades, motivações, projetos futuros e competências académicas anteriores, explicando a diversidade sociocultural crescente desta população estudantil (Almeida, Guisande, Soares, \& Saavedra, 2006; Pope, Mueller, \& Reynolds, 2009; Thorpe, Snell, Hoskins, \& Bryant, 2007).

Um dos aspetos que diferencia os estudantes na sua transição para o ES prende-se com as suas expectativas académicas, entendidas aqui como os objetivos ou aspirações com que os estudantes justificam a sua candidatura e frequência deste nível de ensino. De acordo com a literatura disponível, estas expectativas condicionam os níveis de participação e de envolvimento dos estudantes nas diversas atividades que descrevem o novo contexto académico e social (Almeida, Fernandes, Soares, Vasconcelos, \& Freitas, 2003; Howard, 2005; Konings, Brand-Gruwel, van Merrienboer, \& Broers, 2008; Kuh, Cruce, Shoup, Kinzie, \& Gonyea, 2008; Kuh, Gonyea, \& Williams, 2005; Monteiro, Almeida, Cruz, \& Vasconcelos, 2010). Neste sentido, as expectativas são entendidas como uma variável importante na explicação das trajetórias de transição e adaptação dos estudantes às exigências e desafios do ES (Almeida et al., 2003; Howard, 2005). 
A investigação em torno das expectativas académicas dos estudantes do ES foca diferentes tópicos, nomeadamente a sua evolução ao longo do percurso académico e o seu impacto na aprendizagem e conclusão dos cursos pelos estudantes. Analisando a evolução das expectativas, a literatura sugere que os estudantes ingressam no ES com níveis elevados de expectativas que, muitas vezes, não conseguem concretizar ou manter ao longo do primeiro ano (Almeida et al., 2003; Baker, McNeil, \& Siryk, 1985; Cook \& Leckey, 1999; Credé \& Niehorster, 2012; Smith \& Wertlieb, 2005). Este desencontro entre expectativas iniciais e vivências posteriores aparece associado a dificuldades na integração académica e social, bem como à insatisfação, stress e abandono do ES (Braxton, Vesper, \& Hossler, 1995; Cole, Kennedy, \& Ben-Avie, 2009; Deaño, Alfonso, Conde, Garcia-Señoran, \& Tellado, 2014; Gigliotti, 1987; Jackson, Pancer, Pratt, \& Hunsberger, 2000; Miller, 2005). Por outro lado, a investigação sugere que uma maior congruência entre expectativas e vivências está associada a níveis mais elevados de motivação, persistência e sucesso académico dos estudantes (Deaño et al., 2014; Fernandes \& Almeida, 2005; Neuville et al., 2007; Perera \& Mcllveen, 2014; Robbins, Lauver, Le, Davis, \& Langley, 2004).

Alguma investigação analisa, também, as diferenças nas expectativas académicas segundo algumas variáveis pessoais dos estudantes (Almeida et al., 2006; Alfonso et al., 2013). Por exemplo, alguns autores mencionam que estudantes provenientes de estratos sociais mais desfavorecidos, geralmente também estudantes de primeira geração ou sem antecedentes familiares de frequência do ES, acedem ao ES com ideias menos claras sobre o que é a universidade e que desafios coloca, por vezes formulando expectativas pouco realistas ou, ainda, acreditando pouco nas suas capacidades para os superar (Davies, Slack, Hughes, Mangan, \& Vigurs, 2008; Menon, Saiti, \& Socratous, 2007; Thorpe et al., 2007). Também algumas diferenças têm sido apontadas nas expectativas académicas considerando o sexo dos estudantes. Assim, nalguns estudos, as mulheres revelam expectativas mais elevadas de envolvimento académico, apresentando, aliás, uma taxa superior de conclusão dos seus cursos (Mau \& Bikos, 2000; Mello, 2008; Wells, Seifert, \& Saunders, 2013). Aprofundando as diferenças de género nas diversas dimensões ou tipos de expectativas, os homens apresentam resultados mais elevados nas expectativas relacionadas com o investimento no curso, como 
oportunidade de acesso ao emprego ou a uma carreira profissional (Sax \& Harper, 2007), enquanto as mulheres apresentam resultados mais elevados nas expectativas referentes ao relacionamento interpessoal, seja na procura do apoio dos colegas (Gibson \& Lawrence, 2010; Sax, Bryant, \& Harper, 2005), seja da sua maior disponibilidade para um envolvimento em atividades de voluntariado (Hu \& Wolniak, 2013; Sax \& Harper, 2007). Podendo as estudantes estar mais dependentes emocionalmente da família e do grupo de amigos, a sua participação em programas de mobilidade internacional pode não ser tão valorizada pelas mesmas. Ainda, a participação em atividades científicas com os professores, ou em atividades de carácter político, pode ser superior nos rapazes, pois são atividades que são vistas como requerendo maior autonomia, competição e liderança, tradicionalmente mais associadas aos estudantes homens (Lopez, 2014; Sax, 2009; Sax \& Harper, 2007; Zeldin, Britner, \& Pajares, 2008). Por último, para alguns autores as estudantes estão mais propensas a considerar a opinião e a pressão dos outros (Lopez, 2014; Sax \& Harper, 2007; Zeldin et al., 2008), o que aliás pode contribuir para o seu maior compromisso académico com a frequência e conclusão do curso (Dwyer, Hodson, \& McCloud, 2013; Wells et al., 2013).

$\mathrm{Na}$ linha de outros instrumentos internacionais disponíveis para avaliar as expectativas dos estudantes do ES (Baker et al., 1985; Braxton et al., 1995; Kuh \& Pace, 1998), avançámos na construção e validação de um questionário que pretende abarcar várias dimensões das expectativas académicas. 0 questionário apresenta uma abordagem multidimensional de expectativas académicas, com base em estudos anteriores (Almeida et al., 2003; Fernandes \& Almeida, 2005; Soares, Guisande, Diniz, \& Almeida, 2006), designando-se por Questionário de Perceções Académicas - Expectativas (Almeida, Costa, Alves, Gonçalves, \& Araújo, 2012; Almeida, Deaño, et al., 2012) e integrando 42 itens distribuídos por sete dimensões: Formação para o emprego/carreira; Desenvolvimento pessoal; Mobilidade internacional; Envolvimento político e cidadania; Pressão social; Qualidade da formação; e Interação social.

Neste artigo, estudando uma amostra de estudantes da região da Galiza (Espanha) e do norte de Portugal a frequentarem o primeiro ano do ES, pretendemos analisar eventuais diferenças nas sete dimensões ou tipos de expectativas académicas, considerando o país de origem, se os pais frequentaram ou não o ES e, ainda, o sexo dos estudantes. 


\section{Método}

\section{Participantes}

Neste estudo participaram 717 estudantes do primeiro ano, de Portugal $(n=362)$ e de Espanha $(n=355)$. Não foram considerados estudantes com mais de 25 anos, oscilando a idade entre 17 e 25 anos, tanto em Espanha $(M=19.59$, DP $=1.92)$ como em Portugal $(M=18.56$, $\mathrm{DP}=$ 1.39). Os cursos frequentados pelos estudantes repartiam-se, nos dois países, por duas grandes áreas científicas: cursos de ciências jurídicas, sociais e humanas (52\%) e cursos de ciências e tecnologias (48\%). Face aos objetivos do estudo, balanceou-se a amostra por ambos os países tomando em consideração o sexo dos estudantes e se um ou os dois pais haviam ou não frequentado o Ensino Superior. Face a estes cuidados havidos na constituição da amostra, não se observa uma diferença estatisticamente significativa segundo o país em relação ao sexo dos alunos $\left[\chi^{2}(1)=.006, p=\right.$ .937] e segundo o país em relação aos pais terem ou não frequentado o Ensino Superior $\left[\chi^{2}(1)=.009, p=.923\right]$. Do mesmo modo, não se observa uma associação estatisticamente significativa entre o sexo e a formação superior dos pais $\left[\chi^{2}(1)=3.242, p=.072\right]$.

\section{Instrumento}

As expectativas académicas foram avaliadas através do Questionário de Perceções Académicas - Expectativas (Almeida, Deaño, et al., 2012). Este instrumento avalia as expectativas em sete dimensões: (i) Formação para 0 emprego e carreira, referindo-se às expectativas para obtenção de melhores condições de emprego no acesso ao mercado de trabalho (oito itens; e.g., Ter melhores saídas profissionais no mercado de trabalho); ii) Desenvolvimento pessoal e social, que reflete a valorização do desenvolvimento da autonomia e identidade, autoconfiança e novas experiências de vida (oito itens; e.g., Ganhar confiança nas minhas potencialidades); iii) Mobilidade internacional, que reflete a orientação do estudante para participar em programas de mobilidade estudantil, intercâmbios e estadias internacionais (oito itens, e.g., Participar na mobilidade estudantil, passando algum tempo do curso noutro país); iv) Envolvimento político e cidadania, que reflete a motivação do aluno para discutir a vida política, social e económica do país e contribuir para a 
melhoria da sociedade, exercendo a sua cidadania ativamente (seis itens; e.g., Formar-me como um(a) cidadão(ã) comprometido(a) com os problemas da sociedade atual); (v) Pressão social, que representa a resposta do estudante às expectativas dos outros, nomeadamente da família e grupo de pares, face à sua frequência do ES (quatro itens; e.g., Conseguir corresponder às expectativas dos meus familiares); (vi) Qualidade da formação, que representa as expectativas do estudante face às condições proporcionadas no Ensino Superior para um aprofundamento dos seus conhecimentos (quatro itens; e.g., Aprofundar conhecimentos/matérias na área do meu curso); e, vii) Interação social, relativamente às expectativas do estudante para ter momentos de convivência e diversão, em atividades de interação social no âmbito das relações estabelecidas em contexto universitário (quatro itens; e.g., Ter momentos de convívio e diversão). As respostas dos alunos são fornecidas numa escala de tipo Likert, oscilando entre 1 (Discordo totalmente) e 6 (Concordo totalmente). Nos estudos realizados até ao momento obtiveram-se alfas de Cronbach muito satisfatórios para as dimensões identificadas, oscilando entre .78 e .93, havendo ainda suporte empírico para a diferenciação das sete dimensões com base nos resultados da análise fatorial dos itens (Deaño et al., 2015).

\section{Procedimentos}

Os dados foram recolhidos presencialmente, ocupando cerca de 15 minutos de um tempo letivo cedido pelos professores, previamente contactados. Os estudantes foram informados acerca dos objetivos do estudo e participaram livremente, tendo-Ihes sido garantida a confidencialidade dos resultados. A aplicação do questionário, incluindo uma ficha para obtenção de dados sociodemográficos dos estudantes, ocorreu no início do ano letivo, no $1^{\circ}$ semestre. As análises estatísticas foram realizadas através do programa IBM SPSS (versão 22.0).

\section{Resultados}

$\mathrm{Na}$ tabela 1 apresentamos a média e o desvio-padrão (dentro de parêntesis) dos resultados nas sete dimensões do questionário, considerando os estudantes em função do país, se pelo menos um dos pais tem ou não formação superior (C/FormSup ou S/FormSup, respetivamente) e do sexo. 
Tabela 1 - Resultados nas sete dimensões das expectativas segundo o país, a formação superior dos pais e o sexo dos estudantes

\begin{tabular}{|c|c|c|c|c|c|c|c|c|}
\hline \multirow{3}{*}{ Dimensões } & \multicolumn{4}{|c|}{ Espanha } & \multicolumn{4}{|c|}{ Portugal } \\
\hline & \multicolumn{2}{|c|}{ C/FormSup } & \multicolumn{2}{|c|}{ S/FormSup } & \multicolumn{2}{|c|}{ C/FormSup } & \multicolumn{2}{|c|}{ S/FormSup } \\
\hline & $\begin{array}{c}\text { Masc } \\
(\mathrm{N}=56)\end{array}$ & $\begin{array}{l}\text { Fem } \\
(\mathrm{N}=5)\end{array}$ & $\begin{array}{c}\text { Masc } \\
(\mathrm{N}=99)\end{array}$ & $\begin{array}{c}\text { Fem } \\
(N=144)\end{array}$ & $\begin{array}{c}\text { Masc } \\
(\mathrm{N}=53)\end{array}$ & $\begin{array}{c}\text { Fem } \\
(\mathrm{N}=60)\end{array}$ & $\begin{array}{c}\text { Masc } \\
(\mathrm{N}=104)\end{array}$ & $\begin{array}{c}\text { Fem } \\
(\mathrm{N}=145)\end{array}$ \\
\hline \multirow[t]{2}{*}{ Carreira } & 5.28 & 5.35 & 5.38 & 5.58 & 5.50 & 5.59 & 5.46 & 5.58 \\
\hline & (.78) & (.84) & (.64) & (.44) & $(.59)$ & (.40) & (.50) & (.44) \\
\hline \multirow[t]{2}{*}{ Pessoal } & 4.83 & 5.10 & 4.93 & 5.24 & 5.23 & 5.32 & 5.09 & 5.31 \\
\hline & $(.82)$ & $(.82)$ & $(.66)$ & $(.56)$ & $(.72)$ & $(.53)$ & $(.60)$ & $(.52)$ \\
\hline \multirow[t]{2}{*}{ Mobilidade } & 4.43 & 4.88 & 4.51 & 4.92 & 4.90 & 5.05 & 4.77 & 4.69 \\
\hline & $(1.08)$ & $(.84)$ & (.96) & $(.77)$ & $(.76)$ & (.66) & $(.74)$ & (.68) \\
\hline \multirow[t]{2}{*}{ Cidadania } & 4.13 & 4.47 & 4.19 & 4.71 & 4.75 & 5.11 & 4.57 & 5.15 \\
\hline & $(1.04)$ & $(.86)$ & $(1.00)$ & $(.75)$ & $(.76)$ & (.59) & $(.73)$ & $(.64)$ \\
\hline \multirow{2}{*}{ PresSocial } & 4.45 & 4.69 & 4.56 & 4.72 & 4.60 & 4.64 & 4.71 & 4.55 \\
\hline & $(1.16)$ & $(.92)$ & $(1.10)$ & $(1.02)$ & $(1.07)$ & $(.78)$ & $(.97)$ & $(.98)$ \\
\hline \multirow[t]{2}{*}{ QualForm } & 4.38 & 4.60 & 4.52 & 4.66 & 4.92 & 5.15 & 4.85 & 4.99 \\
\hline & $(.88)$ & $(.73)$ & $(.82)$ & $(.68)$ & $(.68)$ & $(.66)$ & (.62) & $(.57)$ \\
\hline \multirow[t]{2}{*}{ InterSocial } & 4.89 & 4.91 & 4.73 & 4.79 & 4.39 & 4.42 & 4.37 & 4.34 \\
\hline & $(1.08)$ & $(84)$ & $(.97)$ & $(.86)$ & $(1.01)$ & $(80)$ & $(.93)$ & $(.79)$ \\
\hline
\end{tabular}

Analisando as médias apresentadas na tabela 1 , verificamos que a oscilação na dimensão Formação para o emprego e carreira se situa entre 5.28 e 5.59; na dimensão de Desenvolvimento pessoal e social entre 4.83 e 5.32; na dimensão Mobilidade internacional entre 4.43 e 5.05; na dimensão Envolvimento político e cidadania entre 4.13 e 5.15; na dimensão Pressão social entre 4.45 e 4.72; na dimensão Qualidade de formação entre 4.38 e 5.15; e na dimensão Interação social entre 4.34 e 4.91.

Observando os resultados dos estudantes dos dois países, verifica-se que os valores mais baixos nas médias pertencem aos estudantes espanhóis e os mais elevados aos estudantes portugueses, situação que apenas se inverte na dimensão Interação social. Contudo, tomando em consideração que a escala de resposta aos itens oscila entre 1 e 6 , podemos verificar que os estudantes, de uma maneira geral, apresentam expectativas académicas elevadas (com médias superiores a 4.0), em particular nas dimensões Formação para o emprego e carreira e Desenvolvimento pessoal e social.

No sentido de analisarmos o significado estatístico das oscilações observadas nos resultados segundo as três variáveis dos alunos (país, formação académica dos pais e sexo) para as sete dimensões, procedemos a uma análise multivariada da variância ( $F$-Manova: $2 \times 2 \times 2)$. Em primeiro 
lugar, considerando os efeitos secundários ou de interação, não se observou qualquer efeito significativo considerando as três variáveis de agrupamento dos alunos em simultâneo. Da mesma forma, não se observou qualquer efeito significativo combinando a formação académica dos pais e o sexo dos alunos. Um efeito significativo de interação foi, no entanto, observado quando cruzamos o país com o sexo para a dimensão mobilidade $[F(1,716)=7.319$; $p=.007 ;$ eta $\left.^{2}=.007\right]$. Neste caso concreto, verifica-se que em Portugal os resultados praticamente não se diferenciam em função do sexo dos estudantes; contudo, em Espanha, os estudantes do sexo masculino apresentam expectativas mais baixas de mobilidade internacional, quando comparados com os estudantes portugueses ou com as colegas de Espanha do sexo feminino (cf. figura 1).

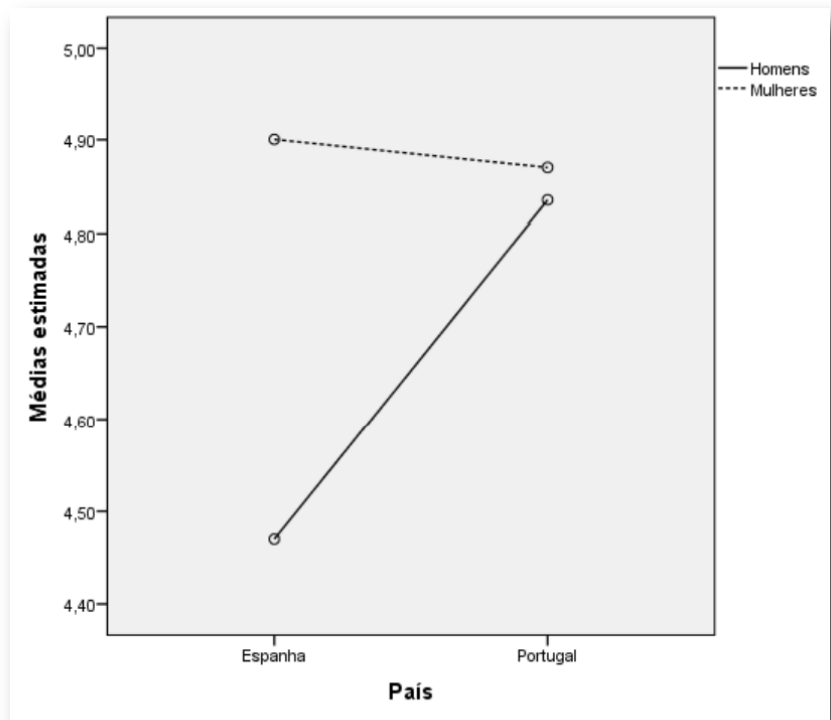

Figura 1 - Interação do país e sexo dos estudantes nos resultados da dimensão 'Mobilidade internacional'

Quando cruzamos o país de origem dos estudantes com as habilitações académicas dos seus pais observa-se um efeito significativo em três das sete dimensões, mais concretamente na Formação para o emprego 
e carreira $\left[F(1,716)=7.547, p=.033 ;\right.$ eta $\left.^{2}=.006\right]$, Desenvolvimento pessoal e social $\left[\mathrm{F}(1,716)=3.915, \mathrm{p}=.048\right.$, eta $\left.{ }^{2}=.005\right]$ e Mobilidade internacional $\left[F(1,716)=5.317, p=.021\right.$, eta $\left.{ }^{2}=.007\right)$. Estas interações apresentam um padrão de resultados relativamente similar, conforme se pode observar nas figuras 2, 3 e 4, nomeadamente nos dois primeiros.

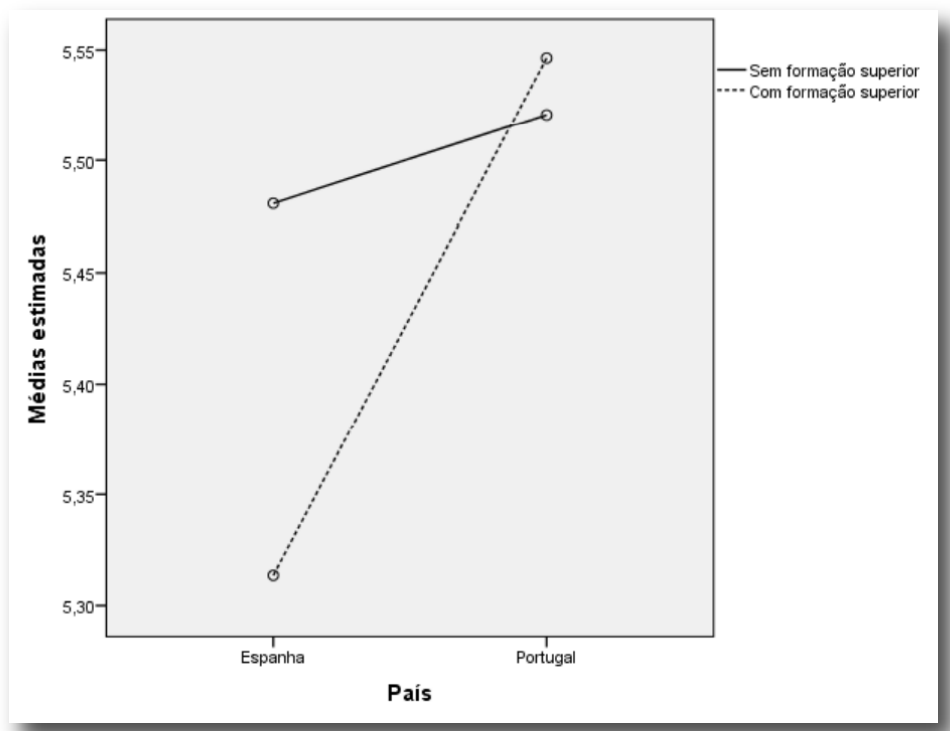

Figura 2 - Interação das variáveis país e habilitações académicas dos pais na dimensão 'Formação para o emprego e carreira' 


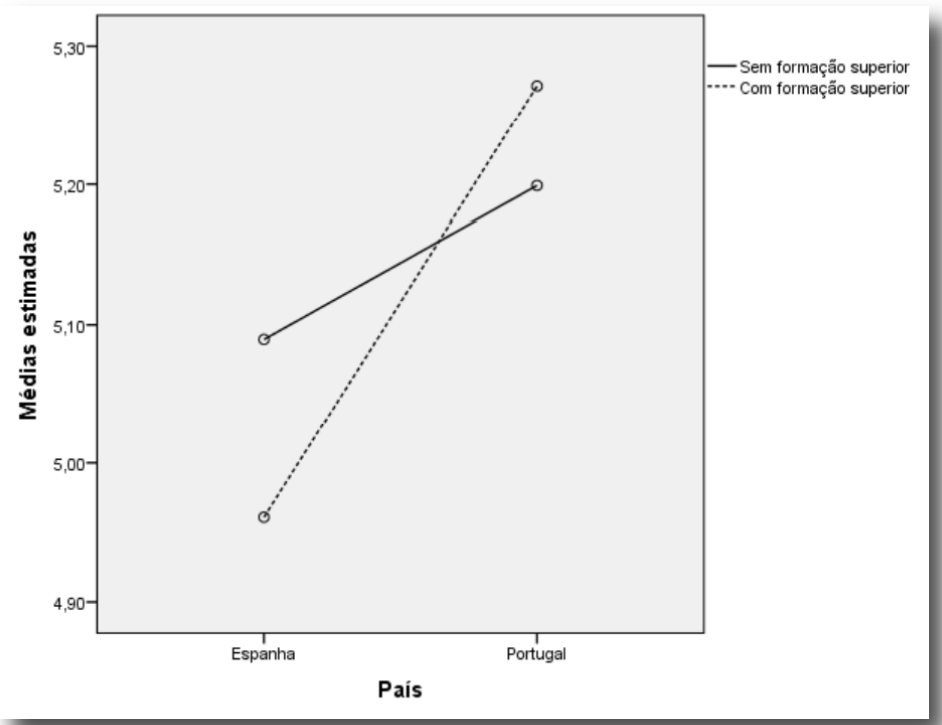

Figura 3 - Interação das variáveis país e habilitações académicas dos pais na dimensão 'Desenvolvimento pessoal e social'

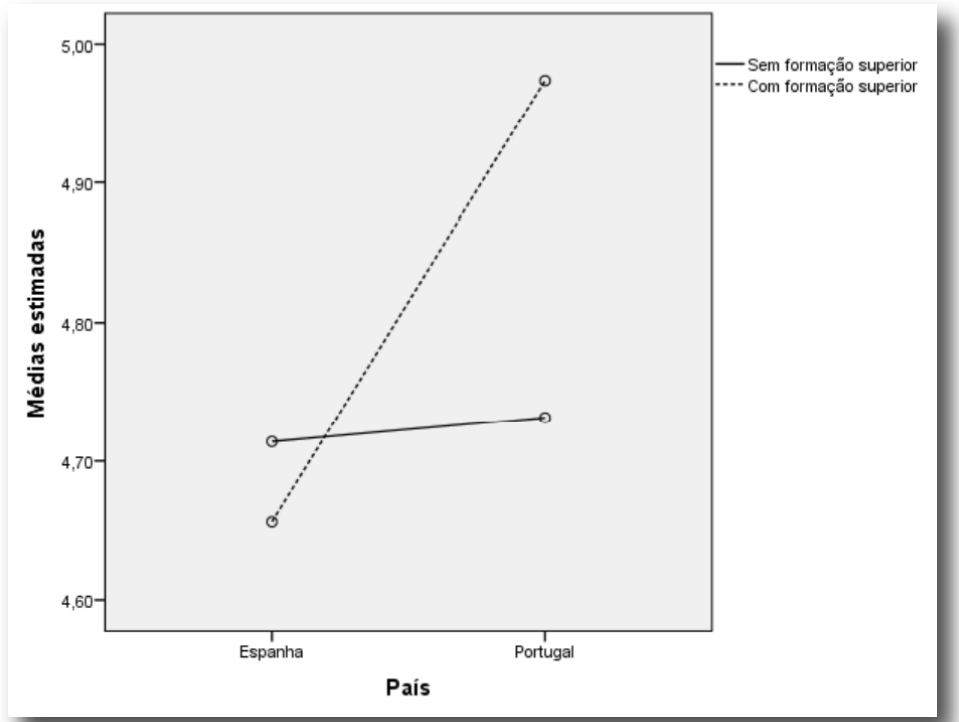

Figura 4 - Interação das variáveis país e habilitações académicas dos pais na dimensão 'Mobilidade internacional' 
Assim, nas dimensões Formação para o emprego e carreira e Desenvolvimento pessoal e social, os estudantes portugueses apresentam expectativas mais elevadas do que os estudantes de Espanha, verificando-se ainda em Portugal que as expectativas dos estudantes cujos pais possuem formação académica superior suplantam ligeiramente as dos colegas cujos pais não possuem essa formação, ocorrendo a situação inversa junto dos estudantes de Espanha. Neste último caso, existe uma maior diferenciação segundo a formação académica dos pais, observando-se expectativas mais elevadas por parte dos estudantes cujos pais não possuem formação académica superior. Na dimensão Mobilidade internacional, os valores não se diferenciam entre os estudantes de Espanha e de Portugal, quando apenas se consideram os estudantes portugueses cujos pais não possuem formação académica superior. Não obstante, para o subgrupo de estudantes portugueses cujos pais possuem formação superior, as suas expectativas de mobilidade internacional são bastante mais elevadas (cf. figura 4).

Reportando-nos agora aos efeitos principais para as variáveis e dimensões em que não se verificaram efeitos de interação, podemos observar diferenças segundo o sexo nas dimensões Formação para a o emprego e carreira $\left[F(1,716)=7.177, p=.008\right.$, eta ${ }^{2}=.010$, Desenvolvimento pessoal $e$ social $\left[F(1,716)=18.536, p=.000\right.$, eta $\left.{ }^{2}=.025\right)$, Envolvimento político e cidadania $\left[F(1,716)=50,090, p=.000\right.$, eta $\left.{ }^{2}=.066\right)$ e Qualidade da formação $\left[F(1,716)=10.362, p=.001\right.$, eta $\left.{ }^{2}=.014\right)$. Em todas estas quatro dimensões, as alunas apresentam expectativas mais elevadas do que os colegas do sexo masculino. Considerando a formação académica dos pais, não se observa qualquer efeito significativo tomando esta variável isoladamente. Por último, considerando o país, para além dos efeitos secundários já descritos, observase um efeito principal desta variável nas dimensões Envolvimento político e cidadania $\left[F(1,716)=65.789, p=.000\right.$, eta $\left.{ }^{2}=.086\right)$, Qualidade da formação $\left[F(1,716)=61.725, \mathrm{p}=.000\right.$, eta $\left.{ }^{2}=.080\right)$ e Interação social $[F(1,716)=$ $37.971, p=.000$, eta2 $=.051)$. Este efeito traduz níveis mais elevados de expectativas por parte dos estudantes portugueses nas dimensões Envolvimento político e cidadania e Qualidade da formação, e níveis mais elevados de expectativas de Interação social por parte dos alunos espanhóis. 


\section{Discussão}

O presente estudo procurou avaliar o impacto do país de origem, formação académica dos pais e sexo dos estudantes nas suas expectativas para o ES. Face a uma situação de progressivo aumento da diversidade sociocultural da população estudantil nas instituições de ES, torna-se ainda mais relevante o estudo dos fatores que diferenciam subgrupos face à qualidade da transição e adaptação ao ES. Neste contexto, elegeram-se as expectativas dos estudantes como variável central a essa transição e adaptação, moderando as trajetórias dos estudantes, à medida que estes se confrontam com as exigências académicas e sociais próprias deste novo contexto de ensino.

Os resultados do presente estudo sugerem que os estudantes, ao ingressarem no ES, apresentam expectativas académicas elevadas, o que tem sido verificado noutros estudos (Almeida et al., 2003; Baker et al., 1985; Cook \& Leckey, 1999; Smith \& Wertlieb, 2005). Neste estudo, estas elevadas expectativas são extensíveis a todas as sete dimensões avaliadas pelo Questionário de Perceções Académicas - Expectativas. Mesmo assim, avaliando-se as respostas da globalidade da amostra e os vários subgrupos de estudantes considerados no estudo, observam-se médias mais elevadas nas dimensões Formação para o emprego e carreira e Desenvolvimento pessoal e social, sendo inversamente mais baixas nas dimensões Interação social, Mobilidade internacional e Envolvimento político e cidadania. Esta diferença traduz distintas prioridades de envolvimento e de adaptação académica dos alunos que ingressam no primeiro ano, onde as vivências adaptativas e eventuais dificuldades associadas ao curso e à identidade podem ser dominantes, podendo as questões da mobilidade e do envolvimento político/cidadania ser preocupações posteriores no percurso académico.

Algumas diferenças nas expectativas académicas dos estudantes foram encontradas em função do país, do sexo e da frequência ou não do ES por parte dos pais. Na dimensão Mobilidade internacional, os estudantes espanhóis do sexo masculino apresentam expectativas mais baixas quando comparados com as estudantes do sexo feminino de Espanha e com os estudantes portugueses dos dois sexos. Em nosso entender, ainda que a mobilidade não pareça assumir-se como uma prioridade em estudantes do 
primeiro ano, este dado parece contrariar os resultados de outros estudos que afirmam um maior envolvimento dos estudantes do sexo masculino em programas de mobilidade estudantil, associados ao seu maior desprendimento socioemocional, liderança e autonomia (Lopez, 2014; Sax, 2009; Sax \& Harper, 2007; Zeldin et al., 2008). Se cruzarmos o país de origem e a existência de, pelo menos, um dos pais com formação superior, verificase um efeito significativo dessa interação nas dimensões Formação para $o$ emprego e carreira e Desenvolvimento pessoal e social, traduzindo níveis particularmente baixos de expectativas por parte dos estudantes espanhóis cujos pais têm formação superior. Por sua vez, na dimensão Mobilidade internacional observam-se expectativas mais elevadas por parte dos estudantes portugueses cujos pais frequentaram o ES. Estes resultados podem estar associados a uma menor tradição de ES em Portugal, que apenas foi sendo progressivamente alterada nas últimas décadas, podendo a origem social dos alunos, em parte associada aos níveis de escolarização dos pais, acabar por assumir um maior peso na diferenciação das expectativas académicas em Portugal. Esta diferença nos estudantes portugueses a favor dos alunos cujos pais possuem formação superior pode acompanhar uma maior quantidade de informação sobre as possibilidades de mobilidade e sua relevância, ao mesmo tempo que, estando a escolaridade dos pais associada ao seu estatuto socioeconómico, serão estudantes com mais recursos económicos familiares suscetíveis de viabilizarem estadias no estrangeiro. Por outro lado, os estudantes espanhóis cujos pais apresentam formação académica superior parecem apresentar níveis mais baixos de expectativas, situação que contraria a investigação na área e justifica análises posteriores.

Os resultados obtidos sugerem, ainda, um efeito da variável sexo na diferenciação das expectativas no que se refere às dimensões Formação para o emprego e a carreira, Desenvolvimento pessoal e social, Envolvimento político e cidadania e Qualidade da formação, apresentando as estudantes do sexo feminino níveis mais elevados de expectativas. Dada a associação positiva entre níveis de expectativas e índices de envolvimento académico (Almeida et al., 2003; Howard, 2005; Konings et al., 2008; Kuh et al., 2008; Kuh et al., 2005), esta diferenciação pode contribuir para as taxas superiores de permanência e de sucesso académico por parte das estudantes do sexo feminino. 


\section{Considerações finais e investigação futura}

Os resultados deste estudo sugerem que os estudantes ingressam no ES com expectativas bastantes elevadas, o que merece das instituições alguma atenção. Algumas vezes, tais expectativas traduzem desconhecimento e ilusões sobre o ES, que seria interessante atenuar aquando do ingresso, sob pena de a desilusão se instalar, dificultando a adaptação dos estudantes ao novo contexto académico. Por outro lado, podendo a frustração das expectativas iniciais conduzir ao desinvestimento e, no limite, ao abandono, seria importante os serviços de ensino e apoio aos estudantes em contexto de ensino secundário e superior estarem atentos a este processo e ajudarem os alunos a construir visões mais realistas sobre o mundo académico, favorecendo a sua transição para o ES.

Defendendo-se um conceito multidimensional de expectativas académicas, e mesmo tendo-se observado padrões elevados de expectativas nas diversas dimensões consideradas nesta avaliação, assiste-se a alguma diferenciação segundo a origem sociocultural dos estudantes, justificando alguma atenção por parte das instituições de ES, cada vez mais preocupadas com a permanência e o sucesso dos seus estudantes. Face a alguma novidade dos resultados obtidos, importa a condução de novos estudos para o aprofundamento das diferenças observadas, dando maior atenção aos cursos ou áreas científicas dos estudantes ou, ainda, ao facto de os alunos frequentarem ou não um curso de primeira escolha e o seu compromisso com a escolha (Araújo, Costa, Casanova, \& Almeida, 2014). Estas variáveis podem ajudar-nos a compreender por que os estudantes espanhóis do sexo masculino provenientes de famílias em que os pais frequentaram o ES apresentam expectativas académicas mais reduzidas, em contraste com o habitualmente encontrado na investigação. Este cuidado na interpretação dos nossos resultados faz tanto mais sentido quando verificamos que, na amostra portuguesa e espanhola, não foi possível considerar os mesmos cursos na formação das duas grandes áreas consideradas (cursos de ciências jurídicas, sociais e humanas, e cursos de ciências e tecnologias).

Por último, importa realizar estudos sequenciais no tempo sobre as expectativas dos alunos que ingressam no ES, procurando aprofundar como estas expectativas acompanham as transformações sociais, por exemplo as crises económicas das famílias e sociedade em geral, ou como influenciam a 
adaptação dos estudantes e o seu sucesso académico (Almeida et al., 2003; Jackson et al., 2000; Smith \& Wertlieb, 2005; Soares et al., 2006). É possível que ocorra algum desinvestimento dos jovens pela frequência do ES face às taxas de desemprego dos diplomados, condicionando assim o seu envolvimento e investimento nas tarefas escolares e relações sociais em contexto universitário. Esta situação, aliás, pode estar, por um lado, mais vincada nos estudantes do sexo masculino, pois a investigação sugere que investem mais no ES sobretudo pelas oportunidades de acesso ao emprego e carreira, e, por outro, pode ocorrer à medida que se avança no curso, pois o tema do desemprego dos diplomados tornar-se-á mais frequente nas conversas entre colegas e com os professores. Uma outra pista de investigação futura será procurar caraterizar perfis de estudantes que ingressam no ES com base na diferenciação das suas expectativas, uma vez que se reconhece que as motivações e objetivos neste acesso não são homogéneos na população estudantil. Tais perfis poderão estar associados a diferenças de trajetórias e investimento no estudo, socialização e desenvolvimento pessoal, bem como aos resultados académicos destes mesmos estudantes.

\section{Notas}

Este trabalho foi apoiado através da atribuição de uma bolsa de investigação de pósdoutoramento à primeira autora (Alexandra M. Araújo), pela Fundação para a Ciência e Tecnologia (SFRH/BPD/85856/2012).

\section{Referências}

Alfonso, S., Deaño, M. D., Rodríguez, A. C., Costa, A. R., Araújo, A. M., \& Almeida, L. S. (2013). Perfiles de expectativas académicas en alumnos españoles y portugueses de Enseñanza Superior. Revista Galego-Portuguesa de Psicoloxía e Educación, 21(1), 125-136.

Almeida, L. S., Costa, A. R., Alves, F., Gonçalves, P., \& Araújo, A. M. (2012). Expectativas académicas dos alunos do Ensino Superior: Construção e validação de uma escala de avaliação. Psicologia, Educação e Cultura, XVI(1), 70-85.

Almeida, L. S., Deaño, M., Araújo, A. M., Costa, A. R., Conde, A., \& Alfonso, S. (2012). Questionário de Perceções Académicas: Versão Expectativas (QPA-E). Braga: Universidade do Minho; Ourense: Universidade de Vigo-Ourense. 
Almeida, L. S., Fernandes, E., Soares, A. P., Vasconcelos, R., \& Freitas, A. C. (2003). Envolvimento académico: Confronto de expectativas e comportamentos em universitários do $1^{\circ}$ ano. Psicologia e Educação, II, 57-70.

Almeida, L. S., Guisande, M. A., Soares, A. P., \& Saavedra, L. (2006). Acesso e sucesso no Ensino Superior em Portugal: Questões de género, origem sócio-cultural e percurso académico dos alunos. Psicologia: Reflexão e Crítica, 19, 507-514. http://dx.doi.org/10.1590/S0102-79722006000300020

Araújo, A. M., Costa, A. R., Casanova, J. R., \& Almeida, L. S. (2014). Questionário de Perceções Académicas - Expectativas: Contributos para a sua validação interna e externa. Revista E-Psi: Revista Eletrónica de Psicologia, Educação e Saúde, 4(1), 156-178.

Baker, R. W., McNeil, O. V., \& Siryk, B. (1985). Expectation and reality in freshman adjustment to college. Journal of Counseling Psychology, 32, 94-103.

Braxton, J., Vesper, N., \& Hossler, D. (1995). Expectations for college and student persistence. Research in Higher Education, 36, 595-612. doi: 10.1007/BF02208833

Cole, J. S., Kennedy, M., \& Ben-Avie, M. (2009). The role of precollege data in assessing and understanding student engagement in college. New Directions for Institutional Research, 141, 55-69. doi: 10.1002/ir.286

Cook, A., \& Leckey, J. (1999). Do expectations meet reality? A survey of changes in first year student opinion. Journal of Further and Higher Education, 23, 157-171. doi: 10.1080/0309877990230201

Credé, M., \& Niehorster, S. (2012). Adjustment to college as measured by the Student Adaptation to College Questionnaire: A quantitative review of its structure and relationships with correlates and consequences. Educational Psychology Review, 24, 133-165. doi: 10.1007/s10648-011-9184-5

Davies, P., Slack, K., Hughes, A., Mangan, J., \& Vigurs, K. (2008). Knowing where to study? Fees, bursaries and fair access. Staffordshire: Staffordshire University.

Deaño, M. D., Alfonso, S. A., Conde, A., Garcia-Señoran, M., \& Tellado, F. (2014). Expectativas universitarias y predicción del rendimiento académico. Revista $E$ Psi: Revista Eletrónica de Psicologia, Educação e Saúde, 4(1), 127-141.

Deaño, M. D, Diniz, A. M., Almeida, L. S., Gil, S. A., Costa, A. R., García-Señorán, M.,... Tellado-González, F. (2015). Propiedades psicométricas del Cuestionario de Percepciones Académicas para la evaluación de las expectativas de los estudiantes de primer año en Enseñanza Superior. Anales de Psicología, 31(1), 280-289. http://dx.doi.org/10.6018/analesps.31.1.161641

Dwyer, R. E., Hodson, R., \& McCloud, L. (2013). Gender, debt, and dropping out of college. Gender \& Society, 27, 30-55. doi: 10.1177/0891243212464906

Fernandes, E. P., \& Almeida, L. S. (2005). Expectativas e vivências académicas: Impacto no rendimento dos alunos do $1^{\circ}$ ano. Psychologica, 40, 267-278.

Gibson, D. E., \& Lawrence, B. S. (2010). Women's and men's career referents: How gender composition and comparison level shape career expectations. Organization Science, 21, 1159-1175. doi:10.1287/orsc.1090.0508 
Gigliotti, R. J. (1987). Expectations, observations, and violations: Comparing their effects on course ratings. Research in Higher Education, 26, 401-415.

Howard, J. A. (2005). Why should we care about student expectations?. In T. E. Miller, B. E. Bender, J. H. Schuh, \& Associates (Eds.), Promoting reasonable expectations: Aligning student and institutional views of the college experience (pp. 10-33). San Francisco, CA: Jossey-Bass.

Hu, S., \& Wolniak, G. C. (2013). College student engagement and early career earnings: Differences by gender, race/ethnicity, and academic preparation. Review of Higher Education, 36, 211-233. doi: 10.1353/rhe.2013.0002

Jackson, L., Pancer, S., Pratt, M., \& Hunsberger, B. (2000). Great expectations: The relation between expectancies and adjustment during the transition to university. Journal of Applied Social Psychology, 30, 2100-2125. doi: 10.1111/j.15591816.2000.tb02427.x

Konings, K. D., Brand-Gruwel, S., van Merrienboer, J. J. G., \& Broers, N. J. (2008). Does a new learning environment come up to students' expectations? A longitudinal study. Journal of Educational Psychology, 100, 535-548. doi: 10.1037/00220663.100.3.535

Kuh, G. D., \& Pace, C. R. (1998). College Student Expectations Questionnaire (2nd ed.). Bloomington, IN: Indiana University, Center for Possecondary Research and Planning.

Kuh, G. D., Cruce, T. M., Shoup, R., Kinzie, J., \& Gonyea, R. M. (2008). Unmasking the effects of student engagement on first-year college grades and persistence. Journal of Higher Education, 79, 540-563. doi: 10.1353/jhe.0.0019

Kuh, G. D., Gonyea, R. M., \& Williams, J. M. (2005). What college students expect from college and what they get. In T. E. Miller, B. E. Bender, J. H. Schuch, \& Associates (Eds.), Promoting reasonable expectations: Aligning student and institutional views of the college experience (pp. 34-64). San Francisco, CA: Jossey-Bass.

Lopez, J. D. (2014). Gender differences in self-efficacy among latino college freshmen. Hispanic Journal of Behavioral Sciences, 36, 95-104. doi: $10.1177 / 0739986313510690$

Mau, W. C., \& Bikos, H. (2000). Educational and vocational aspirations of minority and female students: A longitudinal study. Journal of Counseling \& Development, 78, 186-194. doi: 10.1002/j.1556-6676.2000.tb02577.x

Mello, Z. R. (2008). Gender variation in developmental trajectories of educational and occupational expectations and attainment from adolescence to adulthood. Developmental Psychology, 44, 1069-1080. doi: 10.1037/0012-1649.44.4.1069

Menon, M. E., Saiti, A., \& Socratous, M. (2007). Rationality, information search and choice in higher education. Higher Education, 54(5), 705-721. doi: 10.1007/s10734-006-9019-3

Miller, T. E. (2005). Student persistence and degree attainment. In T. E. Miller, B. E. Bender, \& J. H. Schuh (Eds.), Promoting reasonable expectations: Aligning student and institutional views of the college experience (pp. 122-139). San Francisco: Jossey-Bass. 
Monteiro, S., Almeida, L. S., Cruz, J. F. A., \& Vasconcelos, R. M. (2010). Percepções de alunos de excelência relativamente ao papel dos professores: Um estudo com alunos de engenharia. Revista Portuguesa de Educação, 23(2), 213-236.

Neuville, S., Frenay, M., Schmitz, J., Boudrenghien, G., Noel, B., Wertz, V. (2007). Tinto's theoretical perspective and expectancy-value paradigm: A confrontation to explain freshmen's academic achievement. Psychologica Belgica, 47, 31-50. doi: http://dx.doi.org/10.5334/pb-47-1-31

Perera, H. N., \& Mcllveen, P. (2014). The role of optimism and engagement coping in college adaptation: A career construction model. Journal of Vocational Behavior, 84, 395-404. doi: 10.1016/j.jvb.2014.03.002

Pope, R. L., Mueller, J. A., \& Reynolds, A. L. (2009). Looking back and moving forward: Future directions for diversity research in student affairs. Journal of College Student Development, 50, 640-658. doi: 10.1353/csd.0.0097

Robbins, S. B., Lauver, K., Le, H., Davis, D., \& Langley, R. (2004). Do psychosocial and study skill factors predict college outcomes? A meta-analysis. Psychological Bulletin, 130, 261-288. doi: 10.1037/0033-2909.130.2.261

Sax, L. J. (2009). Gender matters: The variable effect of gender on the student experience. Wiley InterScience, 14(2), 2-10. doi: 10.1002/abc.283

Sax, L. J., Bryant, A. N., \& Harper, C. E. (2005). The differential effects of student-faculty interaction on college outcomes for women and men. Journal of College Student Development, 46, 642-657. doi: 10.1353/csd.2005.0067

Sax, L., \& Harper, C. E. (2007). Origins of the gender gap: Pre-college and college influences on differences between men and women. Research in Higher Education, 48, 669-694. doi: 10.1007/s11162-006-9046-z

Smith, J. S., \& Wertlieb, E. C. (2005). Do first-year college students' expectations align with their first-year experiences? NASPA Journal, 42, 153-174.

Soares, A. P., Guisande, M. A., Diniz, A., \& Almeida, L. S. (2006). Construcción y validación de un modelo multidimensional de ajuste de los jóvenes al contexto universitario. Psicothema, 18, 249-255.

Thorpe, A., Snell, M., Hoskins, S., \& Bryant, J. (2007). False uniqueness: The selfperception of new entrants to higher education in the UK and its implications for access - A pilot study. Higher Education Quarterly, 61(1), 3-22. doi: 10.1111/j.1468-2273.2006.00335.x

Wells, R. S., Seifert, T. A., \& Saunders, D. B. (2013). Gender and realized educational expectations: The roles of social origins and significant others. Research in Higher Education. 54, 599-626. doi: 10.1007/s11162-013-9308-5

Zeldin, A. L., Britner, S. L., \& Pajares, F. (2008). A comparative study of the self-efficacy beliefs of successful men and women in mathematics, science, and technology careers. Journal of Research in Science Teaching, 45, 1036-1058. doi: 10.1002/tea.20195 
PERSONAL AND SOCIOCULTURAL VARIABLES AND THE DIFFERENTIATION OF ACADEMIC EXPECTATIONS: A STUDY WITH HIGHER EDUCATION STUDENTS FROM NORTH PORTUGAL AND GALICIA

\begin{abstract}
Massification of Higher Education in the past decades has resulted in a greater heterogeneity of students. Expectations are a central variable when analysing students' profiles, as they are related to engagement, persistence and academic success. In this study, a questionnaire assessing students' expectations was administered to a sample of 717 first year Portuguese and Spanish students, measuring seven dimensions: i) Training for employment and career development; ii) Personal and social development; iii) Student international mobility; iv) Political engagement and citizenship; v) Social pressure; vi) Quality of education; and vii) Social interaction. Analysis considered the impact of students' country, sex, and parents' education (if parents had a college degree or not). Results evidence that, in general, Spanish students present lower levels of academic expectations compared to Portuguese students. There is also an inverse impact of parents' education on expectations, as Portuguese students whose parents have a college degree present higher expectations, but Spanish students present an inverse pattern of association. Some explanations are presented in the interpretation of these results.
\end{abstract}

Keywords

Higher Education; Academic expectations; First-year students; Sociocultural variables

\title{
VARIABLES PERSONALES Y SOCIOCULTURALES DE DIFERENCIACIÓN DE LAS EXPECTATIVAS ACADÉMICAS: ESTUDIO CON ALUMNOS DE ENSEÑANZA SUPERIOR DEL NORTE DE PORTUGAL Y GALICIA
}

Resumen

Con la expansión del acceso a la Enseñanza Superior (ES), en las últimas décadas asistimos a una mayor heterogeneidad de estudiantes en este nivel 
de enseñanza. Una de las variables importantes a considerar en el análisis de los perfiles de los estudiantes universitarios son sus expectativas académicas, ya que están asociadas a diferentes niveles de implicación, permanencia y éxito académico. Tomando una muestra de 717 estudiantes de $1^{\circ}$ año, portugueses y españoles, se aplicó un cuestionario de expectativas que contempla siete dimensiones: i) Formación empleo/carrera; ii) Desarrollo personal y social; iii) Movilidad internacional; iv) Implicación política y ciudadanía; v) Presión social; vi) Calidad de formación; e vii) Interacción social. Los resultados fueron analizados en función del país, sexo y de ser o no estudiante de $1^{\circ}$ generación (si ninguno de los padres tiene estudios de ES). Los análisis muestran que, de forma general, los estudiantes españoles presentan expectativas más bajas que los estudiantes portugueses. Por otro lado, se observa un efecto en sentido inverso entre ser o no estudiante de primera generación, de modo que los estudiantes portugueses cuyos padres tienen estudios de Enseñanza Superior presentan expectativas más elevadas, produciéndose una situación inversa en los estudiantes españoles. Se avanzan algunas explicaciones en la interpretación de estos datos.

Palabras-clave

Enseñanza Superior; Expectativas académicas; Estudiantes de primer año; Variables socioculturales

Recebido em outubro/2014

Aceite para publicação em abril/2015

i Centro de Investigação em Educação, Instituto de Educação, Universidade do Minho, Portugal. ii Centro de Investigação em Educação Instituto de Educação, Universidade do Minho, Portugal. iii Instituto Superior de Engenharia do Porto, Instituto Politécnico do Porto, Portugal.

iv Departamento de Psicología Evolutiva y Comunicación, Universidade de Vigo-Ourense, Espanha.

v Departamento de Psicología Evolutiva y Comunicación, Universidade de Vigo-Ourense, Espanha.

vi Departamento de Psicología Evolutiva y Comunicación, Universidade de Vigo-Ourense, Espanha.

Toda a correspondência relativa a este artigo deve ser enviada para: Alexandra M. Araújo, Instituto de Educação, Universidade do Minho, Campus de Gualtar, 4710-057 Braga, Portugal. E-mail: alexandra.araujo@ie.uminho.pt 